\title{
Advanced glycation end-products and methionine sulphoxide in skin collagen of patients with type 1 diabetes
}

\author{
Y. Yu • S. R. Thorpe $\cdot$ A. J. Jenkins • J. N. Shaw • \\ M. A. Sochaski • D. McGee • C. E. Aston • \\ T. J. Orchard • N. Silvers • Y. G. Peng • J. A. McKnight • \\ J. W. Baynes • T. J. Lyons • \\ The DCCT/EDIC Research Group
}

Received: 6 September 2005 / Accepted: 15 May 2006 / Published online: 29 August 2006

(C) Springer-Verlag 2006

\begin{abstract}
Aims/hypothesis We determined whether oxidative damage in collagen is increased in (1) patients with diabetes; (2) patients with diabetic complications; and (3) subjects from the Diabetes Control and Complications Trial (DCCT)/Epidemiology of Diabetes Interventions and Complications (EDIC) study, with comparison of subjects from the former standard vs intensive treatment groups 4 years after DCCT completion. Subjects, materials and methods We quantified the early glycation product fructose-lysine, the two AGEs $N^{\varepsilon}$-(carboxymethyl)lysine (CML) and pentosidine, and the oxidised amino acid methionine sulphoxide (MetSO) in skin collagen from 96 patients with type 1 diabetes (taken from three groups: DCCT/EDIC patients and clinic patients from South Carolina and Scotland) and from 78 healthy subjects. Results Fructose-lysine was increased in diabetic patients $(p<0.0001)$, both with or without complications $(p<0.0001)$.
\end{abstract}

Y. Yu • A. J. Jenkins $\cdot$ T. J. Lyons $(\bowtie)$

Section of Endocrinology and Diabetes,

University of Oklahoma Health Sciences Center,

WP1345, Oklahoma City, OK 73104, USA

e-mail: timothy-lyons@ouhsc.edu

S. R. Thorpe $\cdot$ J. N. Shaw $\cdot$ M. A. Sochaski $\cdot$ J. W. Baynes Department of Chemistry and Biochemistry,

University of South Carolina,

Columbia, SC, USA

Y. G. Peng • T. J. Lyons

Division of Endocrinology,

Diabetes and Medical Genetics,

Medical University of South Carolina,

Charleston, SC, USA

D. McGee

Department of Epidemiology,

Medical University of South Carolina,

Charleston, SC, USA
Controlling for $\mathrm{HbA}_{1 \mathrm{c}}$, rates of accumulation of AGEs were higher in diabetic patients than control subjects, regardless of whether the former had complications (CML and pentosidine given as $\log _{\mathrm{e}}[$ pentosidine $]$ ) or not (CML only) (all $p<0.0001)$. MetSO ( $\left.\log _{\mathrm{e}}[\mathrm{MetSO}]\right)$ also accumulated more rapidly in diabetic patients with complications than in controls $(p<0.0001)$, but rates were similar in patients without complications and controls. For all three products, rates of accumulation with age were significantly higher in diabetic patients with complications than in those without (all $p<0.0001$ ). At 4 years after the end of the DCCT, no differences were found between the previous DCCT management groups for fructose-lysine, AGEs or MetSO.

Conclusions/interpretation The findings suggest that in type 1 diabetic patients enhanced oxidative damage to collagen is associated with the presence of vascular complications.

\author{
C. E. Aston \\ General Clinical Research Center, \\ University of Oklahoma Health Sciences Center, \\ Oklahoma City, OK, USA
}

T. J. Orchard $\cdot$ N. Silvers

Department of Epidemiology, University of Pittsburgh,

Pittsburgh, PA, USA

\section{J. A. McKnight}

Metabolic Unit, Western General Hospital,

University of Edinburgh,

Edinburgh, UK

\author{
T. J. Lyons \\ Veterans Administration Medical Center, \\ Oklahoma City, OK, USA
}

The DCCT/EDIC Research Group

The DCCT/EDIC Research Group, Box NDIC/EDIC, Bethesda, MD, USA 
Keywords Collagen - Diabetes complications - Glycation · Methionine sulphoxide $\cdot$ Oxidative stress

$\begin{array}{ll}\text { Abbreviations } \\ \text { CML } & N^{\varepsilon} \text {-(carboxymethyl)lysine } \\ \text { CVD } & \text { cardiovascular disease } \\ \text { DCCT } & \text { the Diabetes Control and Complications Trial } \\ \text { DCCT } & \text { DCCT intensive treatment group } \\ \text { INT } & \\ \text { DCCT } & \text { DCCT standard (conventional) treatment group } \\ \text { STD } & \\ \text { EDIC } & \text { Epidemiology of Diabetes Interventions and } \\ & \text { Complications } \\ \text { ETDRS } & \text { Early Treatment Diabetic Retinopathy Study } \\ \text { FL } & \text { fructose-lysine } \\ \text { Met } & \text { methionine } \\ \text { MetSO } & \text { methionine sulphoxide } \\ \text { MUSC } & \text { Medical University of South Carolina } \\ \text { SC Db } & \begin{array}{l}\text { South Carolina type 1 diabetic } \\ \text { subjects (excluding DCCT/EDIC }\end{array} \\ & \begin{array}{l}\text { subjects) recruited in Charleston, SC } \\ \text { SC }\end{array} \text { South Carolina non-diabetic subjects } \\ \text { non-Db } & \text { recruited in Charleston, SC } \\ \text { SIM-GC/ } & \text { gas chromatography/mass spectrometry } \\ \text { MS } & \text { using selected ion monitoring } \\ \text { UK Db } & \text { type 1 diabetic subjects recruited } \\ & \text { in Edinburgh, UK } \\ \text { UK } & \text { non-diabetic subjects recruited } \\ \text { non-Db } & \text { in Edinburgh, UK }\end{array}$

\section{Introduction}

Modifications of proteins and other macromolecules by glycation, oxidation and 'carbonyl stress' are implicated in the vascular complications of diabetes [1]. In these reactions, the initial glycation product fructose-lysine (FL) is subject to dehydration and rearrangement, forming AGEs such as $N^{\varepsilon}$. (carboxymethyl)lysine (CML) and pentosidine [2]. Much AGE formation, especially in extracellular proteins such as skin collagen, is mediated by reactive carbonyl intermediates involving free radical oxidation [1]. Some end-products, e.g. CML, can be derived from either carbohydrates or lipids [3], and can also form non-oxidatively [4].

The levels of these end-products in a protein depend on the rate of protein turnover (which itself may be influenced by AGE formation), ambient oxidative stress (i.e. the balance between free radicals and antioxidant defences) and ambient substrate (carbohydrate or lipid) levels. Particularly in long-lived structural proteins such as collagen, it may be difficult to estimate the contribution of these variables to the accumulation of AGEs. Levels of FL in collagen are in equilibrium with ambient glucose [5], but
AGEs accumulate steadily with age, and at an accelerated rate in diabetes [6]. Previous studies found that agecorrected skin collagen concentrations of pentosidine and CML, and Maillard-type fluorescence correlated with the severity of microvascular complications in type 1 diabetes [6-9]. However, after normalising for diabetes duration and mean glycaemia, AGE levels were actually lower than expected in diabetes [6]. This suggested that elevated AGEs could be accounted for solely by hyperglycaemia, making it unnecessary to invoke any diabetes-associated increase in free radical oxidation. Nevertheless, in a small sub-analysis, diabetic patients with complications tended to have higher adjusted AGE levels than predicted considering glycaemia, whereas those without complications had lower levels [10]. We hypothesised that these differences might be the result of inter-individual variations in oxidative stress modulating susceptibility to complications. According to this hypothesis, people who experience high oxidative stress will be more susceptible to vascular complications, if they become diabetic.

Methionine sulphoxide (MetSO), a direct oxidation product of methionine, provides a means to assess oxidative stress independent of other chemical modifications [11]. Whereas CML and pentosidine require correction for glycaemia over time to be used as indices of oxidative stress, MetSO, a direct oxidation product, does not. Like AGEs, it accumulates in skin collagen with age, but unlike them, according to a small previous study [12], it is not increased in collagen from diabetic subjects compared to non-diabetic subjects. This again suggested that oxidative stress is not increased, at least in the extracellular compartment, in diabetes. However, that study was too small to test the hypothesis that oxidative stress is higher in complication-prone than in complicationresistant diabetic subjects.

In the present study, we measured FL, CML, pentosidine and MetSO in skin collagen from three groups of complication-prone and complication-resistant type 1 diabetic subjects, and from non-diabetic control subjects. Our goal was to define whether oxidative stress experienced by this extracellular protein is increased in (1) diabetic vs control subjects, (2) diabetic subjects with vs without complications, and (3) standard vs intensive management DCCT/EDIC groups 4 years after DCCT closure.

\section{Subjects, materials and methods}

\section{Human subjects}

Skin biopsies were provided by 96 subjects with type 1 diabetes and 78 non-diabetic control subjects. The diabetic patients came from three separate groups. The largest group $(n=54)$ was from the DCCT/EDIC cohort 
[13]. The original DCCT cohort consisted of 1,441 patients aged 13-40 years with 1-15 years of type 1 diabetes at study entry (1983-1989). Subjects were randomly assigned to standard (STD, $n=730$ ) or intensive (INT, $n=711$ ) treatment for an average of 6.5 years [13] The DCCT was stopped in 1993 because evidence appeared of a powerful beneficial effect of the intensive therapy on microvascular complications, and subjects were invited to join EDIC, a multicentre longitudinal epidemiological investigation [14].

In 1996, a collaborative project between the Medical University of South Carolina (MUSC) and DCCT/EDIC was implemented to identify markers and mechanisms for cardiovascular disease (CVD) in type 1 diabetes. A substudy using skin biopsies to address the role of oxidative stress in complications was implemented at two DCCT/ EDIC centres, Charleston, SC, and Pittsburgh, PA, USA. Of the 54 subjects biopsied in 1997-1999, 26 were from the former INT group and 28 from the STD group.

To increase the study power and relevance, two additional groups of type 1 diabetic subjects were recruited from the diabetes clinics of MUSC $(\mathrm{SC} \mathrm{Db})$ and the Western General Hospital, Edinburgh, UK (UK $\mathrm{Db})$. Apparently healthy non-diabetic control subjects were also recruited at these locations (SC Non-Db and UK Non-Db). The age range of the non-diabetic controls was deliberately broader than that of the diabetic groups to define normal rates of end-product accumulation in skin collagen. The 78 control subjects had no history of diabetes, renal failure or known vascular disease. All studies were approved by the Institutional Review Boards at each centre, and each subject gave written informed consent.

\section{Assessment of complications}

Diabetic subjects were categorised as 'complicated' or 'noncomplicated' based on a review of their medical records before the skin biopsy. 'Complicated' patients had one or more of the following conditions: pre-proliferative or proliferative retinopathy as defined by an Early Treatment Diabetic Retinopathy Study (ETDRS) score $\geq 10$ by previously described DCCT/EDIC methods of retinal photograph analysis [15]; microalbuminuria ( $\geq 40 \mathrm{mg} / 24 \mathrm{~h}$, confirmed on at least two occasions) or heavier proteinuria; or a defined macrovascular event (stroke; myocardial infarction, coronary artery by-pass or angioplasty; intermittent claudication or amputation). 'Non-complicated' patients had minimal retinopathy (ETDRS scores $\leq 3$ ), no proteinuria, and no history of macrovascular events, despite at least 10 years of type 1 diabetes. For the purposes of discussion, the 'complicated' and 'uncomplicated' groups are referred to as being 'complication-prone' and 'complication-resistant'.
Clinical laboratory measures

For DCCT/EDIC subjects, clinical measures were performed as previously described [16] or were obtained from the DCCT/EDIC database. For non-DCCT/EDIC subjects, $\mathrm{HbA}_{1 \mathrm{c}}$ (by HPLC), plasma and urine analyses were carried out in the clinical laboratories of the respective institutional hospital.

\section{Skin biopsy and collagen preparation}

Full-thickness elliptical biopsies $(\sim 0.5 \times 1 \mathrm{~cm})$ were taken from the medial aspect of the buttock (under $2 \%$ lidocaine local anaesthesia), rinsed in PBS and immediately frozen $\left(-70^{\circ} \mathrm{C}\right)$ until collagen extraction was performed as described elsewhere [17]. Briefly, skin was scraped free of adventitious tissue using a scalpel blade and the remaining extracellular protein matrix was extracted sequentially with $1 \mathrm{~mol} / \mathrm{l} \mathrm{NaCl}$, $0.5 \mathrm{~mol} / 1$ acetic acid, and chloroform:methanol (2:1). Each extraction was carried out with gentle mixing $\left(24 \mathrm{~h}, 4^{\circ} \mathrm{C}\right)$. After the final extraction, the insoluble collagen was lyophilised and stored at $-70^{\circ} \mathrm{C}$ until analysed.

Measurement of FL, AGEs and MetSO

Limitations to the biopsy size precluded making all measurements on all samples, but AGEs and MetSO were measured in $\geq 90 \%$ of subjects in each group. FL and CML were quantified by isotope dilution gas chromatography/mass spectrometry using selected ion monitoring (SIM-GC/MS), and pentosidine was quantified by RP-HPLC, as previously described [18]. The concentration of each was normalised to that of the parent amino acid, lysine. Because MetSO is converted to Met during hydrolysis in $\mathrm{HCl}$, MetSO and Met (and $\mathrm{d}_{4}$ MetSO, $\mathrm{d}_{4}$-Met) were measured separately in $0.5-1 \mathrm{mg}$ protein following hydrolysis $\left(4 \mathrm{~h}, 150^{\circ} \mathrm{C}\right)$ in methanesulphonic acid. The acid was removed by ion exchange chromatography, and the remaining amino acids were derivatised with bis-trimethylsilylacetamide for analysis by SIM-GC/MS [19]. MetSO was expressed as a percentage of total Met, i.e. \%MetSO=MetSO/(Met+MetSO).

\section{Data analysis}

For simple comparisons of means and proportions between groups, two-tailed $t$-tests for continuous measures and $\chi^{2}$ analysis for discrete measures were used. The primary dependent variables (FL, CML, pentosidine and MetSO) were analysed using general linear models. The distributions of the residuals from the full model (all terms included) were tested for approximate normality using the Shapiro-Wilks test. Where necessary (MetSO and pentosidine), the dependent variables were log-transformed before 
analysis. For all analyses, diabetic patients with and without complications were analysed as separate groups. The primary hypothesis was that the rate of increase in the dependent variables with age was higher in the diabetic patients with complications than in either those without complications or the non-diabetic controls. This was tested in the general linear model by comparing models with an age effect with those where the age effect was nested within each group, that is, fitting a separate age regression within each group. Independent variables included in the models were age, sex, duration of diabetes and $\mathrm{HbA}_{1 \mathrm{c}}$ levels (except for MetSO). FL was included in models for CML and pentosidine but was found to be equivalent to $\mathrm{HbA}_{1 \mathrm{c}}$ in effect and was removed. Sex and duration were found to have no significant effect and were dropped from the models. The descriptive statistics in the tables are presented as means $\pm \mathrm{SD}$. Values of $p<0.05$ were considered significant. Statistical analyses were performed in SAS (v8) (SAS Institute, Cary, NC, USA).

\section{Results}

Clinical characteristics

Table 1 describes the clinical characteristics of the subjects in this study. Age did not differ significantly between groups. Both diabetes duration and the percentage of smokers were similar between the two DCCT/EDIC groups, and between the SC and UK Db groups, but each of the latter had a longer duration $(p<0.01)$ and a higher proportion of smokers $(p<0.01)$ than the DCCT/EDIC groups. Approximately $25 \%$ of subjects in the previous DCCT INT and UK Db groups had one or more complications, while the fraction of patients with complications was higher (43\%) in the DCCT STD group and was highest (67\%) in the SC Db group. Plasma lipid levels measured at biopsy were comparable among all diabetic and non-diabetic groups.

Glycaemic control was assessed by both long-term (except $\mathrm{SC} \mathrm{Db}$ ) and short-term $\mathrm{HbA}_{1 \mathrm{c}}$ data. Long-term $\mathrm{HbA}_{1 \mathrm{c}}$ levels in the DCCT/EDIC subjects were significantly lower $(p<0.001)$ for patients in the INT vs STD group during the DCCT (1984-1993), but became similar ( $p>0.05)$ after 1994 [14]. For the UK Db patients, long-term 'cumulative' $\mathrm{HbA}_{1 \mathrm{c}}$ was the mean of values obtained at least 3 months apart during the 5 years before the skin biopsy, and in the DCCT/EDIC groups the long-term 'cumulative' $\mathrm{HbA}_{1 \mathrm{c}}$ level was similar to the recent $\mathrm{HbA}_{1 \mathrm{c}}$ levels. Recent $\mathrm{HbA}_{1 \mathrm{c}}$ was therefore used to control the AGE levels for glycaemia. The mean $\mathrm{HbA}_{1 \mathrm{c}}$ at biopsy was not significantly different among the diabetic groups (not considering complications), and was within the normal range in the two non-diabetic groups.

Table 2 summarises the characteristics of the diabetic cohorts according to complication status. Subject age was

Table 1 Characteristics of type 1 diabetic and non-diabetic subjects at biopsy

\begin{tabular}{|c|c|c|c|c|c|c|}
\hline & DCCT INT & DCCT STD & $\mathrm{SC} \mathrm{Db}$ & UK Db & SC Non-Db & UK Non-Db \\
\hline Number of subjects & 26 & 28 & 18 & 24 & 60 & 18 \\
\hline $\operatorname{Sex}(M / F)$ & $10 / 16$ & $17 / 11$ & $3 / 15$ & $15 / 9$ & $30 / 30$ & $13 / 5$ \\
\hline Race (c/aa) & $26 / 0$ & $27 / 1$ & $16 / 2$ & $24 / 0$ & $51 / 9$ & $18 / 0$ \\
\hline Age at biopsy (years) & $37.9 \pm 6.4$ & $40.1 \pm 5.8$ & $37.6 \pm 8.9$ & $40.1 \pm 8.8$ & $42.1 \pm 18.4$ & $32.0 \pm 7.9$ \\
\hline Duration (years) & $17.7 \pm 4.7$ & $18.2 \pm 4.9$ & $24.6 \pm 9.4$ & $22.8 \pm 7.2$ & - & - \\
\hline Smokers (ever) & 3 & 5 & 10 & 13 & 15 & 5 \\
\hline Complications & 6 & 12 & 12 & 6 & - & - \\
\hline Number of subjects & 23 & 22 & 15 & 24 & 55 & 16 \\
\hline Triglycerides (mmol/l) & $1.18 \pm 0.81$ & $0.93 \pm 0.69$ & $1.32 \pm 0.69$ & $1.62 \pm 1.08$ & $1.89 \pm 1.21$ & $1.46 \pm 0.62$ \\
\hline Total chol (mmol/l) & $5.18 \pm 1.01$ & $4.82 \pm 0.91$ & $5.00 \pm 1.01$ & $5.31 \pm 1.11$ & $4.82 \pm 0.93$ & $4.61 \pm 1.21$ \\
\hline LDL-chol (mmol/l) & $3.16 \pm 0.83$ & $2.98 \pm 0.85$ & $2.93 \pm 0.88$ & $2.80 \pm 0.96$ & $2.90 \pm 0.85$ & $2.49 \pm 1.01$ \\
\hline HDL-chol (mmol/l) & $1.48 \pm 0.49$ & $1.42 \pm 0.44$ & $1.40 \pm 0.47$ & $1.35 \pm 0.41$ & $1.11 \pm 0.31$ & $1.22 \pm 0.44$ \\
\hline Number of subjects & 24 & 28 & 12 & 23 & 54 & 13 \\
\hline $\mathrm{HbA}_{1 \mathrm{c}}-\mathrm{DCCT}(\%)$ & $7.6 \pm 0.9$ & $9.0 \pm 1.4$ & - & - & - & - \\
\hline $\mathrm{HbA}_{1 \mathrm{c}}$-EDIC (\%) & $8.6 \pm 1.5$ & $8.5 \pm 1.3$ & - & - & - & - \\
\hline $\mathrm{HbA}_{1 \mathrm{c}}$ at biopsy (\%) & $8.7 \pm 1.7$ & $8.3 \pm 1.5$ & $9.2 \pm 1.9$ & $8.8 \pm 1.3$ & $5.6 \pm 0.5$ & $5.3 \pm 0.4$ \\
\hline $\mathrm{HbA}_{1 \mathrm{c}}$-cumulative $(\%)$ & - & - & - & $8.6 \pm 1.2$ & - & - \\
\hline AGE-modifying drugs ${ }^{\mathrm{a}}$ & \multicolumn{2}{|c|}{$28 / 46(61 \%)$} & \multicolumn{2}{|c|}{$12 / 42(29 \%)$} & \multicolumn{2}{|c|}{$4 / 62(6 \%)$} \\
\hline
\end{tabular}

$\mathrm{UK} \mathrm{Db}$ numbers in italics are for ten subjects only. Values represent mean $\pm \mathrm{SD}$. See text for statistical results

$c$ Caucasian, a a African American, chol cholesterol, complications any manifestation of macrovascular (peripheral, coronary or

cerebrovascular) diseases, and/or pre-proliferative or proliferative retinopathy and/or proteinuria; further details given in Table 2

${ }^{a}$ AGE-modifying drugs include angiotensin-converting enzyme inhibitors, calcium-channel blockers, high-dose vitamin $\mathrm{C}$ and/or E, and statins 
Table 2 Characteristics of type 1 diabetic subjects by complication status

\begin{tabular}{|c|c|c|c|c|c|c|c|c|}
\hline & \multicolumn{2}{|l|}{ DCCT INT } & \multicolumn{2}{|l|}{ DCCT STD } & \multicolumn{2}{|l|}{$\mathrm{SC} \mathrm{Db}$} & \multicolumn{2}{|l|}{ UK Db } \\
\hline & NO Cx (20) & $+\mathrm{Cx}(6)$ & NO Cx (16) & $+\mathrm{Cx}(12)$ & NO Cx (6) & $+\mathrm{Cx}(12)$ & NO Cx (18) & $+\mathrm{Cx}(6)$ \\
\hline $\operatorname{Sex}(M / F)$ & $9 / 11$ & $2 / 4$ & $10 / 6$ & $7 / 5$ & $2 / 4$ & $1 / 11$ & $11 / 7$ & $4 / 2$ \\
\hline Race (c/aa) & $20 / 0$ & $6 / 0$ & $15 / 1$ & $12 / 0$ & $6 / 0$ & $10 / 2$ & $18 / 0$ & $6 / 0$ \\
\hline Age at biopsy (years) & $39.0 \pm 5.8$ & $32.8 \pm 5.0$ & $40.0 \pm 4.4$ & $40.0 \pm 6.9$ & $39.3 \pm 9.3$ & $36.4 \pm 8.8$ & $39.9 \pm 9.3$ & $40.5 \pm 7.1$ \\
\hline Duration (years) & $17.4 \pm 4.7$ & $19.0 \pm 4.1$ & $17.0 \pm 4.4$ & $20.0 \pm 5.0$ & $27.5 \pm 3.5$ & $23.1 \pm 11.4$ & $21.7 \pm 6.8$ & $25.9 \pm 7.9$ \\
\hline Smokers (ever) & 1 & 2 & 1 & 4 & 4 & 6 & 9 & 4 \\
\hline Plasma lipids & NO Cx (19) & $+\mathrm{Cx}(4)$ & NO Cx (13) & $+\mathrm{Cx}(9)$ & NO Cx (6) & $+\mathrm{Cx}(9)$ & NO Cx (18) & $+\mathrm{Cx}(6)$ \\
\hline Triglycerides (mmol/l) & $1.15 \pm 0.89$ & $1.22 \pm 0.41$ & $0.77 \pm 0.38$ & $1.16 \pm 0.97$ & $1.01 \pm 0.52$ & $1.54 \pm 0.78$ & $1.57 \pm 1.15$ & $2.33 \pm 0.45$ \\
\hline Total chol (mmol/l) & $5.10 \pm 0.98$ & $5.59 \pm 1.09$ & $4.87 \pm 0.83$ & $4.77 \pm 1.04$ & $4.74 \pm 1.30$ & $5.18 \pm 0.85$ & $5.31 \pm 1.14$ & $4.01 \pm 2.10$ \\
\hline LDL-chol (mmol/l) & $3.03 \pm 0.73$ & $3.78 \pm 1.11$ & $3.08 \pm 0.88$ & $2.82 \pm 0.91$ & $2.95 \pm 1.14$ & $2.93 \pm 0.52$ & $2.25 \pm 1.50$ & Only 1 value \\
\hline HDL-chol (mmol/l) & $1.53 \pm 0.52$ & $1.27 \pm 0.31$ & $1.42 \pm 0.52$ & $1.40 \pm 0.34$ & $1.32 \pm 0.47$ & $1.53 \pm 0.39$ & $1.35 \pm 0.41$ & Only 1 value \\
\hline $\mathrm{HbA}_{1 \mathrm{c}}(\%)$ & NO Cx (19) & $+\mathrm{Cx}(5)$ & NO Cx (16) & $+\mathrm{Cx}(12)$ & NO Cx (5) & $+\mathrm{Cx}(7)$ & NO Cx (17) & $+\mathrm{Cx}(6)$ \\
\hline DCCT & $7.3 \pm 0.7$ & $8.6 \pm 0.4$ & $8.4 \pm 1.2$ & $9.8 \pm 1.2$ & & & & \\
\hline EDIC & $8.5 \pm 1.4$ & $9.0 \pm 1.7$ & $8.1 \pm 1.0$ & $9.2 \pm 1.4$ & & & & \\
\hline At biopsy & $8.5 \pm 1.5$ & $9.6 \pm 2.1$ & $7.9 \pm 1.1$ & $8.9 \pm 1.8$ & $8.3 \pm 1.0$ & $9.8 \pm 2.2$ & $8.7 \pm 1.1$ & $9.4 \pm 1.7$ \\
\hline Cumulative & & & & & & & $8.3 \pm 0.9$ & $9.6 \pm 1.0$ \\
\hline \multicolumn{9}{|l|}{ Complication status } \\
\hline Proliferative retinopathy & 0 & 0 & 0 & 5 & 0 & 10 & 0 & 6 \\
\hline Microalbuminuria & 0 & 4 & 0 & 5 & 0 & 2 & 0 & 0 \\
\hline \multirow[t]{2}{*}{ Macrovascular } & 0 & 2 & 0 & 5 & 0 & 3 & 0 & 0 \\
\hline & \multicolumn{4}{|c|}{ DCCT INT+STD } & \multicolumn{4}{|c|}{$\mathrm{SC}+\mathrm{UK} \mathrm{Db}$} \\
\hline \multirow[t]{2}{*}{ AGE-modifying drugs } & \multirow{2}{*}{\multicolumn{2}{|c|}{$\begin{array}{l}\text { NO Cx } \\
19 / 32(59 \%)\end{array}$}} & \multicolumn{2}{|l|}{$+\mathrm{Cx}$} & \multicolumn{2}{|c|}{ NO Cx } & \multicolumn{2}{|c|}{$+\mathrm{Cx}$} \\
\hline & & & \multicolumn{2}{|c|}{$9 / 14(64 \%)$} & \multicolumn{2}{|c|}{$7 / 24(29 \%)$} & \multicolumn{2}{|c|}{$5 / 18(28 \%)$} \\
\hline
\end{tabular}

Numbers of subjects are included in parenthesis. Individual subjects may fit more than one complication criterion. UK numbers in italics are for nine subjects. Values represent mean \pm SD. See text for statistical results

Cx Complications

lower $(p<0.05)$ in the patients with complications than in those without complications in the DCCT INT group, but was similar between those with and without complications in the other three groups. Diabetes duration, percentage of smokers and plasma lipids were not statistically different between patients with and without complications in all groups. For all diabetic subjects, $\mathrm{HbA}_{1 \mathrm{c}}$ at biopsy was higher in patients with complications than in those without $(p=0.005)$. Proliferative retinopathy was the most common complication, followed by microalbuminuria.

The proportion of study subjects from each group taking drugs with antioxidant/AGE-modifying potential (angiotensin-converting enzyme inhibitors, calcium-channel blockers, high-dose vitamin $\mathrm{C}$ and/or E, and HMGCoA reductase inhibitors [statins]) at the time of biopsy is shown in Tables 1 and 2. Overall, $61 \%$ of the DCCT group, $29 \%$ of the combined SC and UK diabetic subjects and $6 \%$ of the control subjects were taking potentially AGE-modifying drugs. Considering all the diabetic subjects together, AGE-modifying drugs were taken by $44 \%$ patients with complications and by $46 \%$ without complications (not significant, see Table 2 for further details).
FL levels in skin collagen

Figure 1 demonstrates both the modest rise in collagen glycation with age in non-diabetic individuals and the marked increase, independent of age or complication status, in diabetic subjects. The mean FL in diabetic subjects was 2.3-fold higher than that of the non-diabetic controls $(13.5 \pm 3.5$ vs $5.9 \pm 1.1 \mathrm{mmol} / \mathrm{mol}$ lysine, $p<0.0001)$. The increase of FL among diabetic subjects was little affected by adjustment for age, and was similar to the 1.9-fold increase in mean blood glucose estimated from $\mathrm{HbA}_{1 \mathrm{c}}$ values [20].

Mean FL values were higher in diabetic patients with complications than in those without complications (15.3 \pm 3.7 vs $12.5 \pm 3.0 \mathrm{mmol} / \mathrm{mol}$ lysine, $p=0.0002$ ). This difference also reached statistical significance for patients with and without complications in the DCCT INT group $(p<0.05)$, and for the combined DCCT INT+STD group $(p<0.001)$. Comparing the DCCT INT and STD groups, there was no significant difference in collagen glycation (FL $13.6 \pm 3.6$ vs $14.0 \pm 4.2 \mathrm{mmol} / \mathrm{mol}$ lysine), consistent with their similar $\mathrm{HbA}_{1 \mathrm{c}}$ levels both during EDIC and at biopsy (Table 1). 
Fig. 1 Skin collagen glycation $(F L)$ in diabetic and non-diabetic individuals. FL was measured by SIM-GC/MS in skin collagen from non-diabetic controls $(+, n=47)$, diabetic patients with complications $(\mathbf{m}, n=34)$ and diabetic patients without complications $(\square, n=56)$. The size of the symbol increases with duration of diabetes (range 643 years). FL levels in nondiabetic individuals increased slightly with age (regression line -), but this trend was not observed for diabetic patients. The mean FL level of diabetic subjects was 2.3-fold higher than that of the non-diabetic controls $(13.5 \pm 3.5$ vs $5.9 \pm 1.1 \mathrm{mmol} / \mathrm{mol}$ lysine, $p<0.0001)$. The mean

FL values for all diabetes patients with and without complications were $15.3 \pm 3.7$ and $12.5 \pm 3.0 \mathrm{mmol} / \mathrm{mol}$ lysine, respectively $(p=0.0002)$

\section{AGE accumulation with age}

CML and pentosidine levels in collagen were strongly related to age in both diabetic and non-diabetic populations (Fig. 2), and age-corrected concentrations were significantly higher both in groups of diabetic vs non-diabetic individuals $(p<0.001)$, and in groups of diabetics with vs without complications (CML: $p=0.035$; pentosidine: $p=0.003)$. Controlling for $\mathrm{HbA}_{1 \mathrm{c}}$ to estimate oxidative stress, rates of accumulation of CML and pentosidine (the latter with or without log-transformation to normalise data) with age were higher in diabetic patients with complications than in non-diabetic controls $(p<0.0001)$. For CML only, the rate of accumulation was also higher in diabetic patients without complications than in controls $(p<0.0001)$. Importantly, the rates of accumulation of both AGE products, controlling for $\mathrm{HbA}_{1 \mathrm{c}}$, were higher in diabetic patients with complications than in those without $(p<0.0001)$. Use of collagen FL to control for glycaemia instead of $\mathrm{HbA}_{1 \mathrm{c}}$ yielded the same conclusion. Although AGE products provide only an indirect estimate of oxidative stress, one that requires correction for glycaemia, the data support an increase in the rate of oxidative damage to collagen in diabetic subjects with complications compared with those without complications. The CML data suggested an increase even in patients who do not have complications. These effects were observed despite a higher intake of anti-oxidant, potentially AGE-modifying, drugs in both groups of diabetic subjects. No differences in agecorrected CML and pentosidine were found between the DCCT INT and STD groups, even after correction for
$\mathrm{HbA}_{1 \mathrm{c}}$, nor did the rates of accumulation with age differ after accounting for $\mathrm{HbA}_{1 \mathrm{c}}$.

Collagen MetSO and diabetic complications

The measurement of MetSO in collagen provides a direct measure of oxidative damage in diabetes. As shown in Fig. 3, MetSO accumulated with age in both diabetic subjects and non-diabetic controls. The rate of accumulation was significantly increased in diabetic patients with complications compared with either those without complications or non-diabetic controls (both $p<0.0001$ ). Rates for diabetic patients without complications and control subjects were similar. These results support the presence of increased oxidative stress over time in diabetic patients with complications, but not in those without. MetSO levels did not differ significantly between DCCT INT and STD after controlling for age.

\section{Discussion}

Our goal was to determine associations between oxidative modification of skin collagen and the presence of type 1 diabetes and/or its complications. In diabetes, levels of glycation and AGE products are increased in long-lived proteins such as collagen $[5,6]$. Since AGE formation involves free radical oxidation as well as glycation, AGE levels may be used to assess cumulative oxidative stress if corrected for long-term glycaemia. In this study, two AGE products, CML and pentosidine, were measured. A more 
Fig. 2 Age-dependence of AGEs in skin collagen in diabetic and non-diabetic individuals. CML (a) was measured by SIM-GC/MS and pentosidine (b) by reversed-phase HPLC in skin collagen from non-diabetic controls $(+, n=78)$, diabetic patients with complications (घ, $n=35)$ and diabetic patients without complications

( $\square, n=59$ ). The size of the symbol increases with duration of diabetes. AGE accumulation in skin collagen was strongly related to age in both diabetic and non-diabetic populations as shown by the regression lines (- - non-diabetic, - diabetic with complications, $\cdots$ diabetic without complications). Agecorrected levels of both AGEs were significantly higher in both groups of diabetic vs non-diabetic individuals and in diabetic patients with vs those without complications. Controlling for $\mathrm{HbA}_{1 \mathrm{c}}$, rates of accumulation of AGEs were higher in diabetic patients, whether with complications (CML and pentosidine ( $\log _{\mathrm{e}}$ [pentosidine])) or without (CML only) (all $p<0.0001$ ), than in controls. For both products, rates of accumulation with age were significantly higher in diabetic patients with complications than in those without (all $p<0.0001)$ a

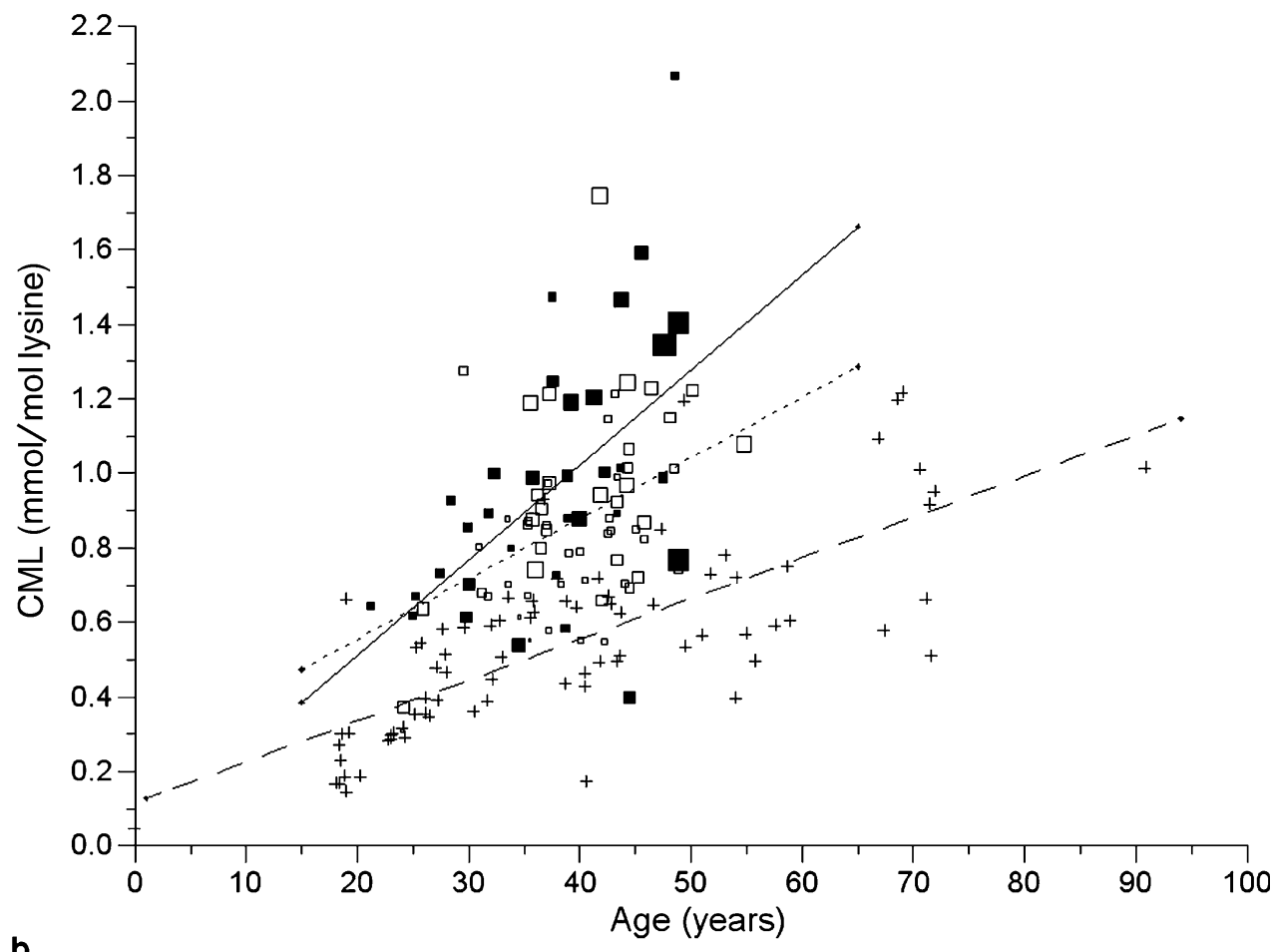

b

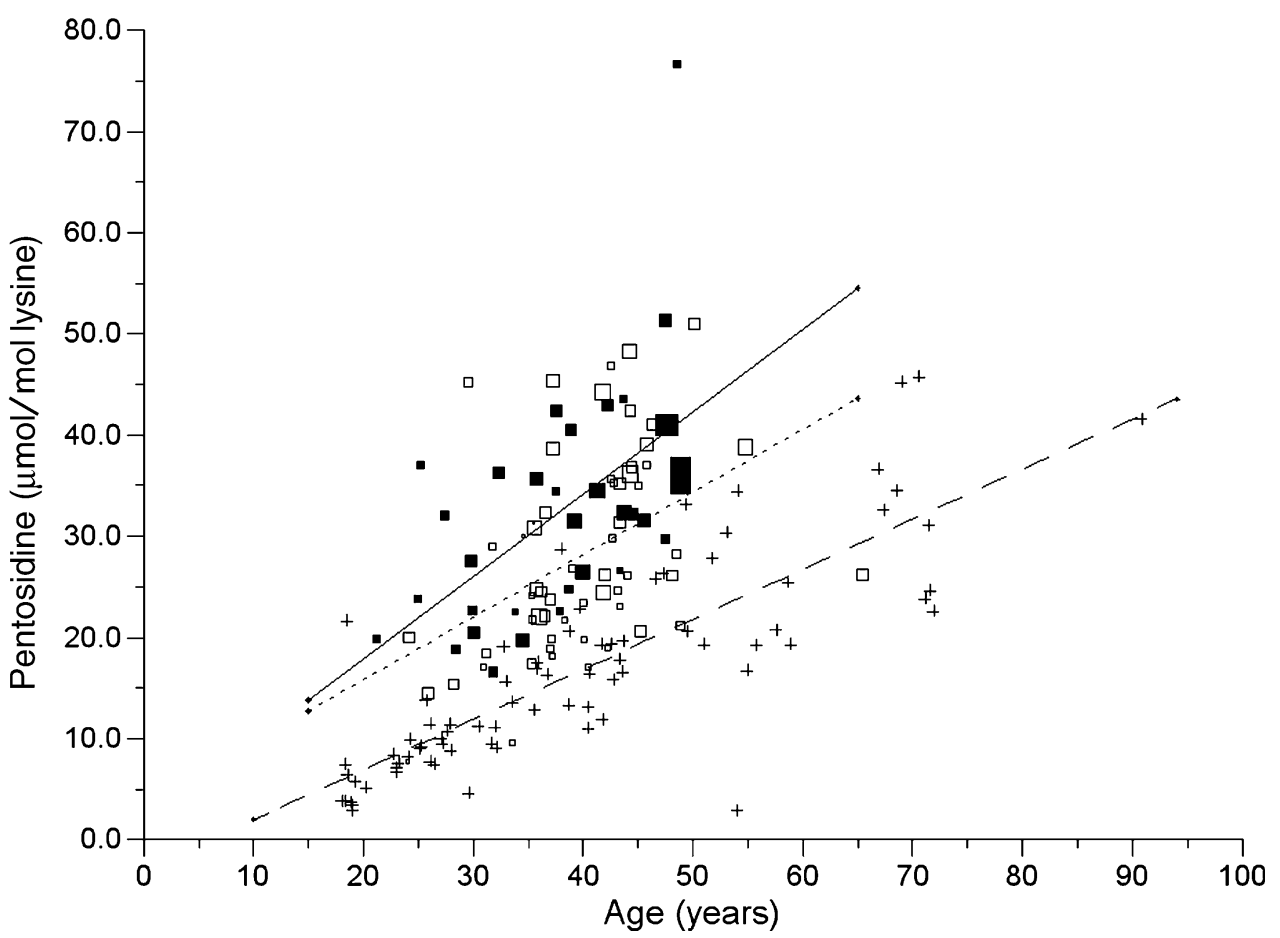

direct measure of cumulative oxidative damage to collagen, MetSO, provides an alternative index of oxidative damage and obviates the need to control for long-term glycaemia.

The diabetic subjects in this study were from the DCCT/ EDIC cohort and clinics in South Carolina and Scotland. The use of these diverse diabetic groups should make our data more generally applicable, with the greatest difference in lifetime diabetes experience likely to be between the former DCCT INT group and all others. For all three products measured, the data strongly support an increased rate of oxidative damage to skin collagen in complicationprone vs complication-resistant diabetic subjects. In the case of CML, but not pentosidine or MetSO, there was evidence of an increased rate of oxidative damage in 
Fig. 3 Age-dependent accumulation of the oxidation product MetSO in skin collagen in diabetic and non-diabetic individuals. MetSO was measured by SIM-GC/MS in non-diabetic subjects $(n=55)$, diabetic patients without complications $(n=47)$, and diabetic patients with complications $(n=33)$. MetSO ( $\left.\log _{\mathrm{e}}[\mathrm{MetSO}]\right)$ accumulated more rapidly in diabetic patients with complications than in controls $(p<0.0001)$, but rates in uncomplicated patients and controls were similar. Key, see Figs. 1 and 2

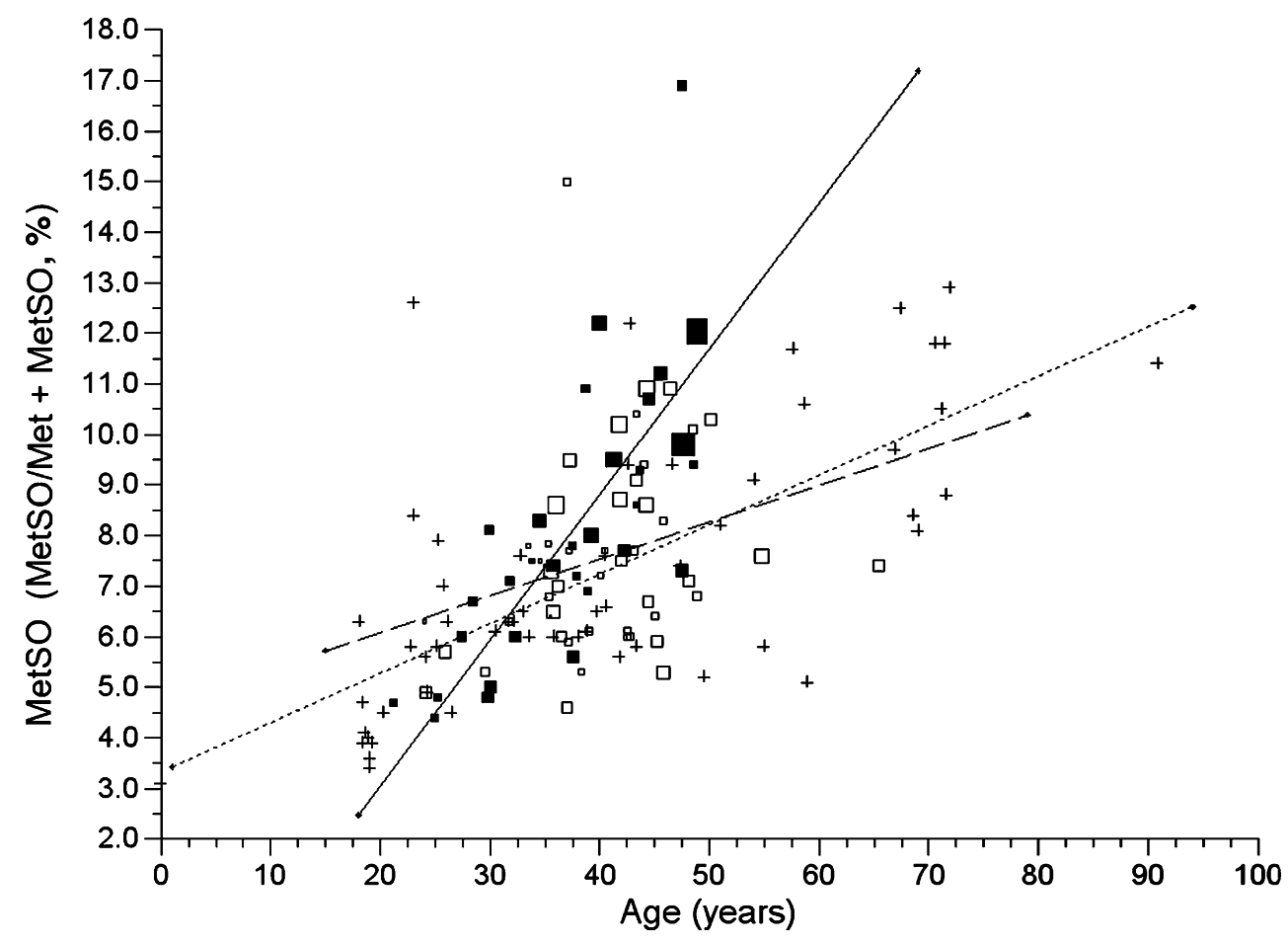

believed to provide a longer term estimate of glycaemia than $\mathrm{HbA}_{1 \mathrm{c}}$, the exact duration to which it relates is unclear. Further, both collagen $\mathrm{FL}$ and $\mathrm{HbA}_{1 \mathrm{c}}$ may reflect longer term glycaemia than theoretically expected, simply because many patients maintain the same degree of glycaemic control for more prolonged periods. It has been suggested that collagen glycation may directly reflect cumulative glycaemia for at least 1 year before measurement [21]. However, our earlier study of type 1 diabetic patients showed that 4 months of intensive therapy decreased skin collagen FL by $25 \%$, while having no effect on levels of collagen AGEs [5].

\section{AGEs, MetSO and complications}

Collagen AGE content increased with age in all groups, and at an accelerated rate in diabetes, confirming earlier studies with both skin collagen and lens proteins [6, 23, 24]. Controlling for age, the mean concentrations of CML and pentosidine were significantly higher in diabetic individuals than in non-diabetics, and in diabetic groups with complications than in those without. This finding was expected, and is consistent with the association of hyperglycaemia with complication status. Controlling for (estimated longterm) glycaemia, using $\mathrm{HbA}_{1 \mathrm{c}}$ (or collagen FL, data not shown), the rates of CML and pentosidine accumulation were significantly greater in complication-prone than complication-resistant subjects, suggesting increased oxidative stress in the complication-prone group. This finding was supported by similar findings with MetSO, in which no correction for glycaemia is needed. 
In contrast, when diabetic patients who were complication-resistant were compared with non-diabetic controls, only CML (and not pentosidine or MetSO) showed evidence of increased oxidative stress. It should be remembered that CML is not only an AGE, but also an 'advanced lipoxidation product' (ALE) [3], perhaps contributing to this distinct association.

The present findings are broadly consistent with our earlier study [6], which did not include a group of patients specifically recruited as complication-prone or complication-resistant. In that study, we concluded that increased collagen CML, pentosidine and fluorescence in type 1 diabetes could be explained entirely by the increase in glycation without invoking increased oxidative stress [6]. However, data from the small number of patients with complications in that study suggested that they might represent a subgroup experiencing higher levels of oxidative damage. The present study strongly supports that contention.

Neither raw nor age-corrected CML, pentosidine, or MetSO levels, nor $\mathrm{HbA}_{1 \mathrm{c}}$-corrected rates of product accumulation over time, differed significantly between subjects from the two former DCCT/EDIC randomisation groups. Concerning absolute levels, this contrasts with the significant differences in collagen AGE levels between patients from the INT vs the STD group at the end of the DCCT, 4 years earlier [21]. Even in that study, however, rates of accumulation (regression line gradients) appeared broadly similar in the two DCCT randomization groups, as they do in our study. The data support the contention that 4 years is a sufficient period of time for equilibration even of the end-products. Given that the recent DCCT report shows that the skin collagen FL and CML levels measured earlier (in 1991-1992) predict the 10-year incidence of retinopathy and nephropathy during EDIC [22], the combination of observations adds further support to the idea that damage resulting from glycation and CML formation may have long-term downstream effects that may explain the 'memory effect' of intensive treatment found during the DCCT.

In contrast to AGEs, which are products of mixed glycation and oxidation, MetSO provides an independent assessment of oxidative damage to protein. MetSO also provides much greater sensitivity than AGEs for assessing protein oxidation with age [12] and avoids variations caused by differing levels of deglycating enzyme activity among individuals [25].

In agreement with our earlier report [12], the present results showed similar rates of collagen MetSO accumulation with age in complication-resistant diabetic and non-diabetic subjects, but a markedly increased rate in complication-prone patients. This suggests that people who have greater than average accumulation of MetSO with age, and who also develop diabetes, may be more susceptible to complications.

Increased rates of accumulation of AGEs and MetSO in patients with complications may reflect enhanced oxidative stress, impaired antioxidant defences, or both. Indirect evidence suggests that a reduced antioxidant capacity could explain the increased MetSO in patients with complications. MetSO can be reduced back to methionine by MetSO reductase, which uses thioredoxin as a co-factor [26]. It has been reported that hyperglycaemia in diabetic animals inhibits thioredoxin function in aortic smooth muscle cells through induction of thioredoxin-interacting protein, an endogenous inhibitor of thioredoxin, leading to increased oxidative stress [27]. This effect is restorable by silencing of the gene encoding thioredoxin-interacting protein and by insulin treatment. Thioredoxin-interacting protein is also induced by glucose incubation in mesangial cells [28]. Therefore, such an impaired thioredoxin system could render patients particularly vulnerable to environmental perturbations, and could make an important contribution to the development of diabetic complications and the increase in MetSO.

Our results suggest that oxidation could be an independent risk factor for vascular complications in diabetes. It would be of interest to see whether this also applies to vascular disease in non-diabetic patients but this is beyond the scope of the present study.

Many drugs, such as angiotensin-converting enzyme inhibitors, calcium-channel blockers, antioxidant vitamins and statins, have potential 'AGE-modifying' activity that could affect our results and interpretation [29-32]. Overall, $61 \%$ of DCCT/EDIC subjects, $29 \%$ of (non-DCCT) South Carolina or Edinburgh diabetic patients and $6 \%$ of control subjects were taking at least one of the potentially antioxidant drugs (Table 1). However, comparing the diabetic patients with and without complications, we found no significant difference in antioxidant consumption within either the DCCT/EDIC group (64 vs 59\%) or the combined $\mathrm{SC} \mathrm{Db}$ and $\mathrm{UK} \mathrm{Db}$ groups (28 vs $25 \%$ ). Combining the diabetic groups, we again did not find any difference in consumption of these medications between patients with or without complications (44 vs 46\%). Thus, our evidence does not support a difference in antioxidant consumption as an explanation for differences in oxidative damage (MetSO) levels between patients with and without complications, but the medications could have disguised a general effect of diabetes in enhancing oxidative damage.

The limitations of this study include its relatively small sample size and the grouping together of several different complications in defining patient categories. Therefore, caution is needed in interpreting these results, and further confirmatory work is required. Our findings are for skin collagen and do not address the effects of diabetes and its 
complications on intracellular oxidative stress [33]. Also, the data do not define the potential effects of localised oxidative stress in complication-prone microenvironments, as suggested by increased ortho- and meta-tyrosine in the diabetic retina [34]. Finally, this cross-sectional study cannot reveal whether the increased rates of AGE and MetSO accumulation are a cause or an effect (or neither) of the complications.

In summary, measurement of skin collagen AGEs and MetSO suggests an increased rate of oxidative damage to collagen in diabetic patients who are prone to vascular complications compared to those who are resistant, but little difference (after accounting for glycaemia as appropriate) between patients with uncomplicated diabetes and nondiabetic control subjects. We did not find significant differences in levels of collagen glycation, AGEs or MetSO between previous DCCT INT and STD groups. Our results suggest that skin collagen AGE (corrected for glycaemic control) and MetSO might be used as surrogate markers to identify diabetic patients who are at high risk of complications, as well as an endpoint for monitoring therapeutic effects.

Acknowledgements This work was supported by NIH Research Grant PO1 HL55782, by Juvenile Diabetes Research Foundation Grants nos 4-1998-272 and 996001, and by a grant from the Diabetes Research and Wellness Foundation, Inc. (Fairfax, VA, USA). It was also supported by a USPHS grant (DK19971) to J. W. Baynes and S. R. Thorpe, by NIH Grant R29 10697 to T. J. Lyons, and by NIH Grants M01-RR-1070 and M01 RR-14467 to the General Clinical Research Centers at MUSC and Oklahoma University Health Sciences Center. The authors acknowledge helpful discussions with V. Monnier and S. Genuth, data analysis by E. Yim and K.-L. Chan, and the technical assistance of W. Li, J. Smith, L. Nichols, M. Brabham and E. Hood.

\section{References}

1. Lyons TJ (2002) Glycation, carbonyl stress, EAGLEs, and the vascular complications of diabetes. Semin Vasc Med 2:175-189

2. Thorpe SR, Lyons TJ, Baynes JW (2000) Glycation and glycoxidation in diabetic vascular disease. In: Keaney JF (ed) Oxidative stress and vascular disease. Kluwer, Norwell, pp 259-283

3. Fu MX, Requena JR, Jenkins AJ, Lyons TJ, Baynes JW, Thorpe SR (1996) The advanced glycation end-product, $N^{\varepsilon}$-(carboxymethyl)lysine, is a product of both lipid peroxidation and glycoxidation reactions. J Biol Chem 271:9982-9986

4. Kasper M, Schieberle P (2005) Labeling studies on the formation pathway of $N^{\varepsilon}$-carboxymethyllysine in Maillard-type reactions. Ann N Y Acad Sci 1043:59-62

5. Lyons TJ, Bailie KE, Dyer DG, Dunn JA, Baynes JW (1991) Decrease in skin collagen glycation with improved glycaemic control in patients with insulin-dependent diabetes mellitus. J Clin Invest 87:1910-1915

6. Dyer DG, Dunn JA, Thorpe SR et al (1993) Accumulation of Maillard reaction products in skin collagen in diabetes and aging. J Clin Invest 9:2463-2469
7. Sell DR, Lapolla A, Odetti P, Fogarty J, Monnier VM (1992) Pentosidine formation in skin correlates with severity of complications in individuals with long-standing IDDM. Diabetes 41:1286-1292

8. Beisswenger PJ, Moore LL, Brinck-Johnsen T, Curphey TJ (1993) Increased collagen-linked pentosidine levels and advanced glycosylation end products in early diabetic nephropathy. J Clin Invest 92:212-217

9. Beisswenger PJ, Moore LL, Curuphey TJ (1993) Relationship between glycaemic control and collagen-linked advanced glycation end products in type 1 diabetes. Diabetes Care 16:689-694

10. McCance DR, Dyer DG, Dunn JA et al (1993) Maillard reaction products and their relation to the complications of diabetes. J Clin Invest 9:2470-2478

11. Onorato JM, Thorpe SR, Baynes JW (1998) Immunohistochemical and ELISA assays for biomarkers of oxidative stress in aging and disease. Ann N Y Acad Sci 854:277-290

12. Wells-Knecht MC, Lyons TJ, McCance DR, Thorpe SR, Baynes JW (1997) Age-dependent accumulation of ortho-tyrosine and methionine sulfoxide in human skin collagen is not increased in diabetes: evidence against a generalised increase in oxidative stress in diabetes. J Clin Invest 100:839-846

13. The Diabetes Care and Complications Trial (DCCT) (1993) The effect of intensive treatment of diabetes on the development and progression of long-term complications in insulin-dependent diabetes mellitus. N Engl J Med 329:977-986

14. Epidemiology of Diabetes Interventions and Complications (EDIC) (1999) Design, implementation, and preliminary results of a long-term follow-up of the Diabetes Control and Complications Trial cohort. Diabetes Care 22:99-111

15. The Diabetes Control and Complications Trial (DCCT) Research Group (1995) The effect of intensive diabetes treatment on the progression of diabetic retinopathy in insulin-dependent diabetes mellitus: the Diabetes Control and Complications Trial. Arch Ophthalmol 113:36-51

16. Jenkins AJ, Lyons TJ, Zheng D et al (2003) Serum lipoproteins in the diabetes control and complications trial/epidemiology of diabetes intervention and complications cohort: associations with gender and glycemia. Diabetes Care 26:810-818

17. Dunn, JA, McCance, DR, Thorpe, SR, Lyons, TJ, Baynes, JW (1991) Age-dependent accumulation of $N^{\varepsilon}$-(carboxymethyl)-lysine and $N^{\varepsilon}$-(carboxymethyl)-hydroxylysine in human skin collagen. Biochemistry 30:1205-1210

18. Dyer DG, Blackledge JA, Thorpe SR Baynes JW (1991) Formation of pentosidine during nonenzymatic browning of proteins by glucose: identification of glucose and other carbohydrates as possible precursors of pentosidine in vivo. J Biol Chem 266:11654-11660

19. Sochaski MA, Jenkins AJ, Lyons TJ, Thorpe SR, Baynes JW (2001) Isotope dilution gas chromatography/mass spectrometry method for the determination of methionine sulfoxide in protein. Anal Chem 73:4662-4667

20. The Diabetes Care and Complications Trial (DCCT) (1987) Feasibility study. Diabetes Care 10:1-19

21. Monnier VM, Bautista O, Kenny DR, et al (1999) Skin collagen glycation, glycoxidation, and crosslinking are lower in subjects with long-term intensive vs conventional therapy of type 1 diabetes: relevance of glycated collagen products $v \mathrm{HbA}_{1 \mathrm{c}}$ as markers of diabetic complications. Diabetes 48:870-880

22. Genuth S, Sun W, Cleary P, et al (2005) Glycation and carboxymethyllysine levels in skin collagen predict the risk of future 10-year progression of diabetic retinopathy and nephropathy in DCCT/EDIC participants with type 1 diabetes. Diabetes 54:3103-3111

23. Ahmed MU, Frye EB, Degenhardt TP, Thorpe SR, Baynes JW (1997) $\mathrm{N}$-epsilon-(carboxyethyl)lysine, a product of the chemical 
modification of proteins by methylglyoxal, increases with age in human lens proteins. Biochem J 324:565-670

24. Degenhardt TP, Thorpe SR, Baynes JW (1998) Chemical modification of proteins by methylglyoxal. Cell Mol Biol 44:1139-1145

25. Szwergold BS, Beisswenger PJ (2003) Enzymatic deglycation-a new paradigm or an epiphenomenon? Biochem Soc Trans 31:1428-1432

26. Hoshi T, Heinemann S (2001) Regulation of cell function by methionine oxidation and reduction. J Physiol 531:1-11

27. Schulze PC, Yoshioka J, Takahashi T, He Z, King GL, Lee RT (2004) Hyperglycaemia promotes oxidative stress through inhibition of thioredoxin function by thioredoxin-interacting protein. J Biol Chem 279:30369-30374

28. Kobayashi T, Uehara S, Ikeda T, Itadani H, Kotani H (2003) Vitamin D3 up-regulated protein-1 regulates collagen expression in mesangial cells. Kidney Int 64:1632-1642

29. Chopra M, Beswick H, Clapperton M, Dargie HJ, Smith WE, McMurray J (1992) Antioxidant effects of angiotensin-converting enzyme (ACE) inhibitors: free radical and oxidant scavenging are sulfhydryl dependent, but lipid peroxidation is inhibited by both sulfhydryl- and nonsulfhydryl-containing ACE inhibitors. J Cardiovasc Pharmacol 19:330-340

30. Miyata T, van Ypersele de Strihou C, Ueda Y, et al (2002) Angiotensin II receptor antagonists and angiotensin-converting enzyme inhibitors lower in vitro the formation of advanced glycation end products: biochemical mechanisms. J Am Soc Nephrol 13:2478-2487

31. Akira K, Amano M, Okajima F, Hashimoto T, Oikawa S (2006) Inhibitory effects of amlodipine and fluvastatin on the deposition of advanced glycation end products in aortic wall of cholesterol and fructose-fed rabbits. Biol Pharm Bull 29:75-81

32. Ng DS (2005) The role of statins in oxidative stress and cardiovascular disease. Curr Drug Targets Cardiovasc Haematol Disord 5:165-175

33. Brownlee M (2001) Biochemistry and molecular cell biology of diabetic complications. Nature 414:813-820

34. Pennathur S, Ido Y, Heller JI et al (2005) Reactive carbonyls and polyunsaturated fatty acids produce a hydroxyl radical-like species: a potential pathway for oxidative damage of retinal proteins in diabetes. J Biol Chem 280:22706-22714 